\section{Cireulaire du 20 octobre 1906, concernant les instructions relatives à l'emploi du béton armé}

En présence du développement que prennent les applications du béton armé aux travaux publics, il est nécessaire de faire connaître aux ingénieurs les conditions générales, moyennant lesquelles les constructions faites avec cette matière nouvelle présentent les mêmes caractères de stabilité, et offrent au public les mêmes garanties de sécurité, que celles qui sont édifiées avec les matériaux anciennement éprouvés.

La question a fait l'abjet de longues études, et de recherches expérimentales, qui se sont poursuivies durant trois années, pour aboutir au dépôt d'un rapport dont le Conseil général des Ponts et Chaussées a été saisi, et qu'il a renvoyé à une Commission spéciale composée d'inspecteurs généraux.

Sur le rapport de cette Commission, en date du 20 juillet 1906 dont une copie est annexée à la présente circulaire, et après une discussion approfondie, le Conseil général des Ponts et Chaussées a adopté un projet d'instructions applicables à l'emploi du béton armé dans les ouvrages dépendant du ministère des Travaux publics.

Conformément aux décisions du Conseil, le ministre a approuvé ces instructions dont on trouvera plus loin le texte.

Elles sont conformes à l'état actuel de nos connaissances en la matière, mais seront sans.doute à reprendre, lorsque l'expérience des chantiers et des laboratoires, et une plus longue carrière du béton armé, auront fourni, en ce qui le concerne, des données plus certaines que celles que l'on possède aujourd'hui.

Les explications qui suivent ont pour objet de préciser, en tant que de besoin, le sens et la portée de ces instructions.

\section{I. - Données à admettre dans la préparation des projets.}

\section{A. - Surcharges.}

Article premer. - Les ponts en béton armé sont établis de manière à pouvoir supporter les surcharges verticales, et les actions du vent, imposées aux ponts métalliques, de même destination, par le règlement du 29 août 1891 .

Art. 2. - Les combles en béton armé sont, sauf exception justifiée, soumis, au point de vue des surcharges, au règlement du 17 février 1903 , relatif aux halles métalliques des chemins de fer.

Art. 3. - Les planchers et autres parties des bâtiments, les conduites sous pression, et tous autres ouvrages intéressant la sécurité publique, sont calculés en vue des plus grandes surcharges qu'ils auront à supporter en service.

\section{B. - Limites de travail ou de fatigue.}

ArT. 4. - La limite de fatigue a la compression du béton armé, à admettre dans les calculs de résistance des ouvrages, ne doit pas dépasser les ving huit centièmes $(0,28)$ de la résistance a l'écrasement acquise par le béton non armé de même composition, après quatre-vingt-dix-jours de prise.

La valeur de cette résistance, mesurée sur des cubes de vingt centimètres de côté, est spécifiée au devis de chaque projet.

Arr. 5. - Lorsque le béton est fretté, ou, lorsque les armatures transversales ou obliques qu'il porte sont disposées de manière à s'opposer plus ou moins efficacement à son gonflement sous linfluence de la compression longitudinale qu'il supporte, la lintite de fatigue à la compression prévue à l'article précédent peut être majorée dans une mesure plus ou moins large suivant le volume et le degré d'efficacité des armatures transversales, sans que la nouvelle limite puisse, quel que soit le pourcentage du métal employé, dépasser les soixante centièmes de la résistance à l'écrasement du béton non armé, telle qu'elle est définie à l'article 4 .
Art. 6. - La limite de fatigue au cisallement, au gissement longitudinal du béton sur lui-même, et à son adhérence sur le métal des armatures, est prévue égale à dix centièmes de celle spécifiée à l'article 4 pour la limite de fatigue à la compression.

Art. 7. - La limite de fatigue, tant à l'extension qu'à la compression, qui ne peut pas être dépassée pour le métal employé aux armatures, est la moitié de sa limite apparente d'élasticité, telle qu'elle est définie au devis de chaque projet. Toutefois, pour les pièces supportant des chocs, ou soumises à des efforts de sens alternés, telles que les hourdis, cette limite est réduite aux quarante centièmes, au lieu de moitié de la limite apparente d'élasticité.

ArT. 8. - Pour les pièces soumises à des efforts très variables, les limites de travail, ci-dessus définies, sont abaissées d'autant plus que les variations sont plus grandes, sans que la diminution exigée puisse être de plus de 25 pour 100 .

Les limites de travail sont également abaissées pour les pièces soumises à des causes de fatigue, ou d'affaiblissement, dont les calculs de résistance n'ont pas tenu compte, notamment à des actions dynamiques, comme celles que supportent les pièces placées direclement sous les rails des voies ferrées.

\section{II. - Calculs de résistance.}

ArT. 9. - Dans les calculs de résistance des ouvrages en béton armé, il est tenu compte non-seulement des plus grandes forces extérieures, y compris les actions du vent et de la neige, que ces ouvrages pourront avoir à supporter, mais aussi des effets thermiques, et de ceux du retrait du béton, toutes les fois qu'il ne s'agira pas d'ouvrages librement dilatables dans le sens théorique du mot, ou de ceux que l'expérience permet de regarder approximativement comme tels.

ArT. 1o. - Les calculs de résistance sont faits selon des méthodes scientifiques, appuyées sur les données expérimentales, et non par des procédés empiriques. Ils sont déduits soit, des principes de la résistance des matériaux, soit de principes offrant au moins les mêmes garanties d'exactitude.

Art I1. - La résistance du béton à l'extension est mise en compte dans le calcul des déformations. Mais, pour déterminer la fatigue locale dans une section quelconque, cette résistance est regardée comme nulle dans la section.

ART. 1 2. - Pour les pièces comprimées, on s'assure qu'elles ne sont pas exposées à flamber. Toutefois, on peut s'en dispenser pour les pièces dont l'élancement (rapport de la hauteur à la plus faible dimension transversale) est inférieure à 20 , et dont la fatigue à la compression ne dépasse pas la limite définie par l'article 4 .

ArT. 13. - Le devis doit indiquer les qualités et dosage des matières entrant dant la composition du béton; quant à la proportion d'eau à employer pour le gâchage, elle doit être surveillée avec soin, et strictement suffisante pour donner au béton la plasticité nécessaire pour le bon enrobage des armatures et le remplissage de tous les vides.

\section{III. - Exécution des travaux.}

ArT. 14. - Les coffrages, ainsi que l'arrimage des armatures présentent une rigidité suffisante pour résister sans déformation sensible aux charges et aux chocs qu'ils sont exposés à subir pendant l'exécution du travail, et jusqu'au décoffrage et aux décintrements inclusivement.

Art, 15-Sauf dans le cas exceptionnel où le ciment est coulé, il est toujours à prise lente, et damé avec le plus grand soin par couches dont l'épaisseur est en rapport avec les dimensions des matériaux employés et les intervalles des armatures, et ne dépasse pas $0^{m} 05$ après damage, à moins qu'on n'emploie des cailloux.

Art. 16. - Les distances des armatures entre elles et aux parois des coffrages sont telles qu'elles permettent le parfai 
damage du béton et son serrage contre les armatures. Ces de nières distances, même quand on n'emploie que du mortier sans gravier, ni cailloux, doivent toujours être d'au moins i 5 à 20 millimètres, de façon à mettre les armatures à l'abri des intempéries.

Art. i 7. - Lorsqu'on emploie, pour les armatures, des fers profilés, et non des barres rondes, on prend des dispositions spéciales pour que leur enrobage se fasse parfaitement sur tout leur périmètre, et notamment dans les angles rentrants.

Art. I8. - Lorsque l'exécution d'une pièce a été interrompue, ce qu'on évite autant que possible, on nettoie à vif et on mouille l'ancien béton assez longtemps pour qu'il soit bien imbibé avant d'ètre mis en contact avec du béton frais.

Art. 19. - En temps de gelée, le travail est interrompu si l'on ne dispose pas de moyens efficaces pour en prévenir les effets nuisibles.

A la reprise du travail, on opère la démolition de tout ce qui a subi les atteintes de la gelée, puis on procède comme il est dit à l'article précédent.

Art. 20. - Pendant quinze jours au moins après son exécution, l'on entretient dans le béton l'humidité nécessaire pour en assurer la prise dans de bonnes conditions.

Le décoffrage et le décintrement sont faits sans chocs, par des efforts purement statiques, et seulement après que le béton a acquis la résistance nécessaire pour supporter sans dommage les efforts auxquel il est soumis.

\section{IV. - Épreuves des ouvrages.}

Art. 2 I. - Les ouvrages en béton armé qui intéressent la sécurité publique sont éprouvés avant d'être mis en service. Les conditions des épreuves, ainsi que les délais de mise en service sont insérés au cahier des charges. Les flèches maxima que les ouvrages ne doivent pas dépasser sont aussi, du. moins autant qu'on le peut, insérées au cahier des charges.

L'âge que le béton doit avoir au moment des épreuves est de même fixé par le cahier des charges. Il est d'au moins go jours pour les grands ouvrages, de $4^{5}$ jours pour les ouvrages de moyenne importance, et de 30 jours pour les planchers.

ART. 22. - Les ingénieurs profitent des épreuves pour faire non seulement toutes les mesures de déformation ou de vérification des conditions du cahier des charges, mais aussi, autant que possible, celles qui peuvent intéresser la science de l'ingénieur.

Pour les ouvrages de quelque importance, on emploie des appareils enregistreurs.

Art. 23. - Les ponts en béton armé sont éprouvés dè la manière prescrite pour les ponts métalliques par le règlement du 29 août 189 I.

S'il paraît convenable d'apporter certaines dérogations aux prescriptions de ce règlement, elles doivent être justifiées $\epsilon t$ insérées au cahier des charges.

ArT. 24. - Les combles sont éprouvés de la manière prescrites par le règlement du 17 février 1903 , sauf dérogations à justifier.

ArT, 25. - Les planchers sont soumis à une épreuve consistant à appliquer les charges et surcharges prévues, soit à la totalité du plancher, soit au moins à une travée entière.

Les. surcharges doivent rester en place pendant 24 heures au moins. Les fièches ne doivent plus augmenter au bout de I 5 heures.

Le Minisire des Travaux pubilics, des Postes et des Télégraphes,

Louis Barthou.

\section{RAPPORT DE LA COMMISSION}

nommée par le Conseil Général des Ponts et Chaussées dans sa séance du 15 mars $1906{ }^{(1)}$

\section{Emploi du Béton armé.}

Ce rapport pourra être très bref, parce que la Commission a fait son possible pour que les projets d'instruction et de circulaire qu'elle a préparés forment un tout qui puisse suffire aux ingénieurs, et, par conséquent, au Conseil.

Il sera seulement indiqué dans quel ordre d'idées on a cru devoir remanier les projets de règlements et de circulaire préparés par la Commission du ciment armé, et l'on s'empressera de dire que les différences portent plutôt sur la forme que sur le fond, tout en n'étant pas sans importance.

En tout cas, la Commission n'a cru devoir rien faire sans avoir pris l'avis des deux principaux représentants actuels de la Commission du ciment armé : son rapporteur, M. l'inspecteur général Considère, et son président, depuis la retraite de $\mathrm{M}$. le président Lorieux : M. l'ingénieur en chef Résal.

Cette commission, en effer, a aciompli une œuvre considérable, à laquelle elle a cónsacré quatre années, et dont les pièces mises entre les mains des membres du Conseil, à savoir: les projets de règlements et de circulaires préparés par elle, et le magistral rapport du plus qualifié en la matière, M. l'inspecteur général Considère, ne donnent, malgré leur importance, qu'une idée imparfaite. Il faut, en outre, avoir examiné les procèsverbaux des expériences de longue haleine, auxquelles la Commission s'est livréc, avec le concours de $M$. l'ingénieur Mesnager et du laboratoire de l'école des Ponts et Chaussées, pour pouvoir apprécier toute l'étendue et la portée de son œuvre. Aussi, convenait-il de n'y toucher qu'avec la plus grande réserve, et en ayant son avis. C'est dans cette pensée que la Commission a cherché à remplir la mission que le Conseil lui a fait l'honneur de lui confier, mission fort délicate; car si le béton armé est de plus en plus apprécié dans ses effets, il est encore bien imparfaitement connu dans ses causes. Plus on y réfléchit, plus on sent qu'il y a là nombre de phénomènes qui demeurent obscurs. Dans ces conditions, il n'est pas aisé d'arriver à la précision désirable dans les instructions à donner aux ingénieurs, tout en ne les entravant pas dans la voie du progrès qui reste ouverte. C'est sans doute le sentiment de ces difficultếs qui a arrêté la Commission du ciment pendant plusieurs années. C'est lui aussi qui doit servir d'excuse à la Commission du béton armé pour les quelques semaines de réflexion qu'elle a prises.

Cette Commission a cherché à aller vite. Peu de jours après sa désignation, elle s'est réunie. Elle a tenu deux séances auxquelles ont été convoqués MM. Considère et Résal. Là, on a discuté contradictoirement tous les articles du projet de règlement de la Commission du ciment armé, ainsi que le projet de circulaire, et le rapport de $M$. Considère qui l'aciompagne.

Puis la Commission s'est ajournée, en chargeant le soussigné de préparer ses propositions.

Dans l'intervalle, le soussigné a reçu, au nom de la minorité de la Commission du ciment armé, un projet de règlement signé par $M$. l'ingénieur en chef Rabut et $M$. l'ingénieur Mesnager, deux membres très qualifiés, eux aussi, de ladite Commission.

Leurs observations portaient sur deux points : l'un relatif à la valeur du coefficient d'élasticité du béton, l'autre tendant à ce que les prescriptions contenues dans le projet de règlement, et relatives aux calculs de résistance des matériaux, soient de beaucoup abrégées, et réduites à quelques indications générales, de façon à éviter tout ce qui pourrait tendre à restreindre, en cette matière, la liberté scientifique des ingénieurs, sauf à reporter dans la circulaire les explications ou les conseils que l'on pourrait juger utile de leur donner.

(1) Commission composée de : MM. Maurice Lfivy, Inspecteur général, de $1^{\text {ro }}$ classe, membre de l'Institut, Président et Rapporteur: de PréauDEAU, Vétrirlart, inspecteúrs généraux de $2^{e}$ classe. 
Sur ce dernier point, tout le monde a fini par tomber d'accord; tel fut aussi le sentiment du Conseil général des ponts et chaus. sées, dans la séance où l'affaire est venue en discussion, et a été, après un échange d'observations, renvoyée à la Commission que le soussigné a l'honneur de présider.

A l'appui de leurs observations sur le coefficient d'élasticité, MM. Rabut et Mesnager ont joint les résultats d'une série d'expériences faites par $M$. Mesnager, expériences qui ont été naturellement versées au dossier, ainsi que diverses auires pièces, notamment un projet de règlement préparé par ces ingénieurs.

Des expériences dont il s'agit, il ressort que, jusqu'à un effort de 60 kilogrammes par centimètre carré, le béton expérimenté par eux, et composé de 300 kilogrammes de ciment de Portland pour 400 litres de sable et 800 litres de gravier, a un coefficient d'élasticité environ égal au 1/10 du coefficient d'élasticité de l'acier. C'est aussi ce qui ressort à peu près des expériences de M. le professeur Bach, de Stuttgart, et de celles qui avaient été entreprises, en France, dès les débuts du ciment armé, à la demande du regretté directeur des phares, Bourdelles.

C'est ainsi, muni d'une part des explications échangées pendant les deux premières séances avec les deux représentants de la majorité de la Commission du cinjent armé, MM. Considère et Résal, des explications fournies au nom de ceux de la minorité de la Commission, que le soussigné s'est mis à l'œuvre pour préparer, non sans de fréquents scrupules, les projets d'instructions et de circulaire que la Commission actuelle a l'honneur de soumettre à l'examen du Conseil.

A ce mot « règlement», employé par la Commission du ciment armé, a été substitué le mot a instructions » qui, tout en ayant le même caractère obligatoire pour les ingénieurs, s'annonce comme moins permanent. Il convient, en effet, de prévoir que l'expérience des chantiers, comme celles des labo. ratoires et comme la théorie, pourront modifier les vues qu'on a actuellement sur le ciment armé, et, par suite, amener à faire des retouches aux prescriptions actuelles.

En principe, on a cherché à condenser ces instructions en un petit nombre d'articles, brefs et précis.

Elles sont divisées en quatre parties:

I. Données à admettre dans les projets relatifs au béton armé.

II. Calculs de résistance (à appuyer sur ces données).

III. Exécution des travaux.

IV. Epreuves des ouvrages.

I. Données à admettre. - Ces données comprennent deux parties distinctes: les surcharges et les coefficients de travail.

Il n'y a rien à dire relativement aux surcharges. Elles sont les mêmes pour les ouvrages en ciment armé que pour leurs similaires en d'autres matières.

La fatigue à la compression du ciment armé a été admise égalè aux $28 / 100$ de la résistance à l'écrasement du béton non armé de même composition, après go jours de confection, cette résistance étant mesurée sur un cube de $\mathrm{O}^{\mathrm{m}} 20$ de cồté.

La Commission du ciment armé, dans son projet de règle. ment, n'avait indiqué la fatigue maxima à admettre que pour trois espèces de ciments, qui sont formées de 800 litres de gravier, 400 litres de sable, avec respectivement les trois dosages: 300,350 et 400 kilogrammes de Portland.

Elle a trouvé pour ces bétons, respectivement, les résistances suivantes en kilogrammes par $\mathrm{cm}^{2}$.

Au bout de 28 jours : Io7, r 20 et r 33 kilogrammes.

Au bout de 90 jours: 160,180 et 200 kilogrammes.

Elle admet dans son réglement les limites de fatigue ciaprès: $45,52,58$ kilogrammes.

La règle proposée par la Commission donne: 44,$8 ; 50,4 ;$ 56 kilogrammes, c'est.à-dire sensiblement les mêmes chiffres.

La Commission est donc d'accord avec celle du ciment armé, et sa formule a l'avantage de s'étendre ä d'autres ciments de compositions très variables qui peuvent être employćs dans la pratique.
Mais, ce n'est pas sans hésitation que la Commission a été suivie sur ce point. Ce taux de fatigue des $28 / 100$ de la résistance après go jours est élevé, et beaucoup plus élẹvé que les chiffres similaires admis dans d'autres règlements, notamment dans les règlements allemands ou suisses. Là où la Commission admet une fatigue de 5 I kilogrammes, on n'admeturait guère que 30 à 35 kilogrammes.

MM. Résal et Considère, au nom de la Commission du ciment armé, ont insisté pour le maintien des chiffres proposés par cette Commission, après une longue discussion en présence des représentants de l'Industrie qui ont fait partie de la Commission. Ils ont fait valoir que les chiffres admis sont ceux cou ramment usités dans la pratique, et que l'industrie ne pourrait se contenter de chiffres notablement moindres. $M$. Considère a fait connaître, depuis, que les réglements étrangers sont déjà anciens, eu égard aux rapides progrès accomplis par le béton armé, qu'ils donnent lieu, au point de vue spécial dont il s'agit, à des réclamations de la part des constructeurs, et que, vraisemblablement, soit par tolérance, soit par une modification aux prescriptions existantes, on sera amené à élever notablement le taux de fatigue admis à une époque où l'on n'avait pas encorc, en matière de béton armé, l'expérience acquise depuis.

On verra, d'ailleurs, que les données acceptées pour les calculs de résistance sont de nature à rassurer sur les valeurs élevées adoptées pour les taux de farigue aux articles 4 c 5 .

Ce dernier article permet de majorer le taux normal de fatigue, admis à l'article 4 .

11 constitue une innovation, relativement aux instructions étrangères qu'il a été donné à la Commission de consulter, en ce qu'il encouragera les constructeurs à porter leur attention, non seulement sur les armatures longitudinales, mais aussi sur les armatures transversales, qui ont une influence considérable sur la solidité de ce genre de constructions. Il mérite d'être conservé. Il est formulé sous forme générale dans les instructions. Le commentaire qu'y donne la circulairè avec le coefficient de majoration $\left(1+m, \frac{V}{V}\right)$ guidera les ingénieurs dans l'adoption du taux de la majoration suivant le cas. Par une sorte d'interprétation rapide, on peut, avec une suffisante approximation, passer des cas spécitiés dans la circulaire à des cas différents pour le choix du coefficient $m^{\prime}$ qui, seul, reste à l'appréciation des ingénieurs.

II. Calculs de résistance. - On voit que les instructions se bornent à quelques prescriptions générales qui laissent aux ingénieurs la plus absolue liberté dans les méthodes de calcul qu'ils croiron: devoir employer, sous la seule réserve de ne pas substituer les méthodes empiriques des spécialistes aux méthodes plus sûres tirées de la résistance des matériaux, ou de la théorie de l'élasticité. Mais, comme d'autre part il est à notre connaissance que beaucoup d'ingénieurs seraient très heureux d'avoir quelques indications qui puissent leur servir de guides dans ces calculs, nouveaux pour beaucoup d'entre eux, la circulaire a cherché à donner à ce désir la satisfaction la plus large possible, tout en y faisant remarquer que les formules, et même les methodes indiquées, n'ont aucun caractère obligatoire, et que toutes autres méthodes, pourvu qu'elles soient rationnelles, seront admises par l'Administration.

On doit insister, non sur les formules contenues dans la circulaire, et qui sont déduites des principes de la résistance des matériaux relatifs aux pièces à sections hétérogènes, mais sur l'une des données qui y est indiquée ou conseillée et qui, comme celle signalée plus haut à loccasion de l'article 5, innove relativement à ce qui existe, et est de nature, comme on l'a fait pressentir plus haut. à atténuer sensiblement ce que le taux élevé de fatigue à la compression du béton admis aux articles 4 et 5 peut avoir de hardi. Il s'agit d'un nombre que l'on admet dans les calculs de résistance pour exprimer l'équivalence, à section égale, entre le béton et l'armature. Dans les formules de la plupart des auteurs, français et étrangers, on admet que, dans la compression d'un prisme armé, chaque 
centimètre carré de l'armature longitudinale supporte une part de charge $m$ fois plus grande que ne le ferait un centimètre carré de béton occupant la même place.

Theoriquement, le nombre $m$ serait le rapport entre le module d'élasticité du métal et celui du béton. MM. Rabut et Mesnager demandaient que ce nombre fût pris égal à ro. En Suisse et en Allemagne, comme aussi d'après les auteurs français et belges, on adopte de préférence la valeur 15 .

Il est vraisemblable qu'avec ce dernier chiffre on attribue souvent au métal une infuence plus grande que la réalité, et au béton une influence trop faible, de sorte que celui-ci supportera, en réalité, une fatigue plus grande que celle que supposent les calculs.

L'innovation de la circulaire consiste à proposer pour ce nombre $m$, non pas une valeur immuable, telle que ro ou 15 , mais une valeur dépendant à la fois des dîspositions de l'armature longitudinale et de celles des armatures transversales ou obliques qui les solidarisent. On ałmet que le nombre $m$ peut ainsi varier, suivant que les dispositions des armatures sont plus ou moins combinées, entre un minimum de 8 et un maximum de 15 .

Cette manière de faire semble très rationnelle théoriquement, outre qu'elle s'ajoute aux prescriptions de l'article 5 des instructions, pour inciter les praticiens à bien étudier les dispositions combinées des armatures longitudinales et transversales.

La Commission s'est assurée, d'ailleurs, qu'on arrive ainsi à un coefficient de sécurité bien plus constant qu'avec les ouvrages calculés dans l'hypothèse de la constance de $m$, ce qui diminue sensiblement le danger pouvant résulter du coefficient de fatigue élevé qu'on a adopté aux articles 4 et 5 des instructions.

Pour bien comprendre le genre de vérification qui a été poursuivi, il convient de préciser le sens qu'on attache a l'expression : coefficient de sécurité.

Si l'on suppose une colonne en béton armé où, d'après les calculs de résistance, le béton travaille à raison de 50 kilogrammes par $\mathrm{cm}^{2}$, tandis qu'un cube du même béton non armé se romprait après 90 jours sous une charge de 200 kilogrammes par $\mathrm{cm}^{2}$, on dira que le coefficient de sécurité est 4 . Mais (et cette observation s'applique aussi aux ouvrages autres que ceux en béton armé), ce n'est là qu'un coefficient conventionnel, le seul, en général, qu'on puisse fixer, et dont il faut, par suite, se contenter dans la pratique. Le vrai coefficient de sécurité ne pourrait s'obtenir qu'en rompant, non plus un cube de béton non armé, mais en rompant la colonne elle-même. Or, il est probable que, mème abstraction faite du flambage que l'on suppose combattu, la colonne se romprait sous une charge autre que le cube de béton. Si elle n'était fas armée, elle se romprait sous une charge un peu plus faible, en raison des points faibles que comporte un ouvrage de plus grandes dimensions, et moins bien soigné, dans ses moindres détails, qu'un échantillon cubique de $o^{\mathbf{m}_{2}}$ o de côté. Grâce à l'armature, et c'est là son but, ou, du moins, l'un d'eux, il se peut que la colonne supporte, avant rupture, une charge égale ou supérieure à celle qu'a pu supporter l'échantillon cubique.

Dans le premier cas, le coefficient de sécurité conventionnellement rapporté à cet échantillon serait trompeur et illusoire. Dans le second, au contraire, i] serait très sûr, puisqu'il ne pourrait qu'être égal ou inférieur au coefficient de sécurité réel.

En tous cas, ce dernier ne peut s'obtenir que par destruction directe de l'ouvrage considéré. Ce coefficient réel a été déterminé, sur un prisme de béton armé, à base carrée de $o^{m_{2}} 5$ de côté et de I mètre de hauteur, portant diverses armatures, à l'aide d'expériences de rupture très précises de $M$. le professeur Bach. Aux charges de rupture expérimentalement déterminées, on a comparé les fatigues qui résulteraient:

- De l'emploi des formules de résistance avec un coefficient $m$ constant et égal à i 5 ;

$2^{\circ}$ De l'emploi des formules avec un coefficient $m$ variable entre 8 et i 5 , selon les règles indiquées dans la circulaire, et en faisant, d'après ces règles, des interpolations à vue, et avec les majorations de la fatigue admises par l'article 5 des instructions pour l'emploi des coefficients de majoration:

$$
1+m^{\prime} \frac{V^{\prime}}{V}
$$

le coefficient $m$ ' étant également obtenu, dans chaque cas, d'après les règles indiquées dans la circulaire.

Voici les données expérimentales et les résultats obtenus:

Section du prisme: $\Omega=25 \times 25=625 \mathrm{~cm}^{2}$.

Volume $V^{\prime}$ des ligatures : $V^{\prime}=62 \mathrm{~cm}^{3} 645$.

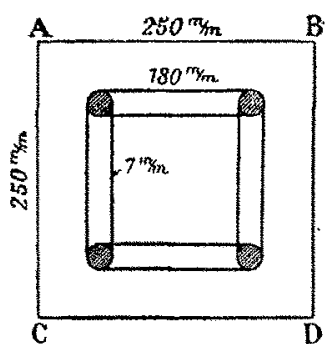

Les prismes essayés ont une section carrée $A B C D$ de 250 millimètres de côté. Ils sont armés de cinq tiges eloignées d'axe en axe de 180 millimètres et ayant des diamètres $d$ variables de 15 à $30 \mathrm{mi}$ llimètres.

Ces tiges longitudinales sont réunies deux à deux par des tiges formant ligatures transversales doubles, suivant les quatre côtés d'un carré.

Toutes ces tiges ont 7 millimètres de diamètre.

L'écartement de ces armatures transversales dans le sens de l'axe du prisme varie de $\mathrm{om}_{2} 5$ à $\mathrm{o}^{\mathrm{m}} 0625$.

\section{TABleaU I}

\begin{tabular}{|c|c|c|c|c|}
\hline 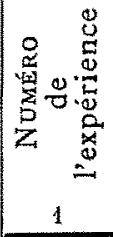 & $\begin{array}{c}\text { Diamètre } d \\
\text { des } \\
\text { armatures } \\
\text { longitudinales } \\
\text { mm. } \\
2\end{array}$ & $\begin{array}{c}\text { ECARTEMENT } \\
\text { des } \\
\text { armatures } \\
\text { transversales } \\
\mathrm{cm} . \\
3 \\
\end{array}$ & $\begin{array}{c}\text { VaLeur } \\
\text { moyenne } \\
\text { de la charge } \\
\text { de rupture } \\
\text { kgs par } \mathrm{cm}^{2} \\
4\end{array}$ & $\begin{array}{c}\text { Secrron } \\
\text { des } \\
\text { armatures } \\
\text { longitudinales } \\
\omega=4 \frac{\pi d^{2}}{400} \mathrm{~cm}^{2} \\
5\end{array}$ \\
\hline $\begin{array}{l}1 \\
2 \\
3 \\
4 \\
5\end{array}$ & $\begin{array}{l}15 \\
15 \\
15 \\
20 \\
30\end{array}$ & $\begin{array}{r}25,00 \\
12,00 \\
6,00 \\
25,00 \\
25,00\end{array}$ & $\begin{array}{l}168 \\
177 \\
205 \\
170 \\
190\end{array}$ & $\begin{array}{r}7,1 \\
7,1 \\
7,1 \\
12,6 \\
28,3\end{array}$ \\
\hline
\end{tabular}

De plus, la charge de rupture du prisme non armé a été trouvée de........................ $14.1 \mathrm{~kg} .95$ et celle d'un mètre cube de ce béton de....... $175 \mathrm{~kg} .95$

En supposant $m=15$, et appelant $R_{\mathrm{b}}$ la fatigue admise pour le beton, la charge totale $N$ que pourrait supporter le beton serait :

$$
N=R_{\mathrm{b}}(625+15 \mathrm{w})
$$

En prenant $R_{\mathrm{b}}=35 \mathrm{kilog}$, ce qui serait conforme aux insiructions allemandes, on trouve:

$$
N=35(625+15 \omega) \text {. }
$$

Le résumé des cinq séries d'expériences est donné par le

\begin{tabular}{|c|c|c|c|c|c|c|}
\hline 焉 & $\frac{15 \omega}{\mathrm{cm}^{2}}$ & $\begin{array}{c}625+15 \omega \\
3\end{array}$ & $\begin{array}{c}N \\
\text { kgs } \\
4\end{array}$ & 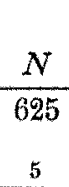 & $\begin{array}{c}\text { Charice } \\
\text { de } \\
\text { rupture } \\
B .\end{array}$ & $\begin{array}{c}\text { Confry- } \\
\text { crent } \\
\text { de } \\
\text { securité } \\
\text { effectif } \\
7\end{array}$ \\
\hline $\begin{array}{l}1 \\
2 \\
3 \\
4 \\
5\end{array}$ & $\begin{array}{l}106 \\
106 \\
106 \\
189 \\
424\end{array}$ & $\begin{array}{r}731 \\
731 \\
731 \\
814 \\
1049\end{array}$ & $\begin{array}{l}25.585 \\
25.585 \\
25.585 \\
28.499 \\
36.715\end{array}$ & $\begin{array}{l}40,9 \\
40,9 \\
40,9 \\
48,6 \\
58,7\end{array}$ & $\begin{array}{l}168 \\
177 \\
205 \\
170 \\
190\end{array}$ & $\begin{array}{l}4,1 \\
4,3 \\
5,0 \\
3,7 \\
3,2\end{array}$ \\
\hline
\end{tabular}
tableau 11 .

TABLEAU $\cdot$ II

La colonne 5 donne la charge théorique par centimètre carré que supporte le béton de la colonne, la colonne 6 , reproduction 
TableaU III

\begin{tabular}{|c|c|c|c|c|c|c|c|c|c|c|}
\hline $\begin{array}{c}\text { Nos } \\
1\end{array}$ & $m$ & $\begin{array}{c}m \omega \\
\mathrm{cm}^{2} \\
3\end{array}$ & $\mid \begin{array}{c}625+m \omega \\
\end{array}$ & $\begin{array}{c}\text { ÉCARTEMENT } \\
\text { des armatures } \\
\text { transversales } \\
5\end{array}$ & $m^{\prime}$ & $\frac{\dot{V}}{V}$ & $\begin{array}{c}R_{b}=50 \\
1+m^{\prime} \frac{V}{V} \\
8\end{array}$ & $\begin{array}{c}N \\
\mathrm{~kg} \\
9\end{array}$ & $\begin{array}{c}\frac{N}{625} \\
10\end{array}$ & $\begin{array}{l}\text { Comfricrent } \\
\text { de sécurité } \\
\text { effectif } \\
11\end{array}$ \\
\hline $\begin{array}{l}1 \\
2 \\
3 \\
4 \\
5 \\
5\end{array}$ & $\begin{array}{r}10 \\
12 \\
15 \\
9 \\
8\end{array}$ & $\begin{array}{r}71 \\
85 \\
106 \\
113 \\
226\end{array}$ & $\begin{array}{l}696 \\
710 \\
731 \\
738 \\
851\end{array}$ & $\begin{array}{l}0 \text { m20 } \\
0,125 \\
0,0625 \\
0,25 \\
0,25\end{array}$ & $\begin{array}{r}8 \\
12 \\
15 \\
8 \\
8\end{array}$ & $\begin{array}{l}0,00401 \\
0,00802 \\
0,01604 \\
0,00404 \\
0,00400\end{array}$ & $\begin{array}{l}51,6 \\
5,4,8 \\
62,0 \\
51,6 \\
51,6\end{array}$ & $\begin{array}{r}35,913 \\
38,908 \\
55,322 \\
48,080 \\
43,911\end{array}$ & $\begin{array}{l}57,4 \\
62,3 \\
72,5 \\
60,9 \\
70,2\end{array}$ & $\begin{array}{l}2,9 \\
2,8 \\
2,8 \\
2,8 \\
2,7\end{array}$ \\
\hline
\end{tabular}

de celle 4 du tableau I, donne les charges de rupture effectives. correspondantes. En divisant les chiffres de la colonne 6 par ceux correspondants de la colonne 5 , on aura, dans chaque cas, le coefficient de sécurité effectif. On voit qu'il a des variations très considérables. Il varie entre 5 et 3,2 , ce qui indique que la formule [A], c'est-à-dire l'hypothèse de la constance de $m$, peut conduire à de sérieux mécomptes.

Faisant à présent les mêmes calculs en supposant $m$ variable, et suivant les règles indiquées dans la circulaire, on est amené, par des interpolations, à donner à $m$ les valeurs du tableau III.

D'autre part, on admet pour le béton, en nombre rond, d'après l'article 4 des instructions, une fatigue de 50 kilog., au lieu de celle de 35 admise ci-dessus, et en vertu de l'article 5, on majore cette fatigue d'après les coefficients de majoration:

$$
1+m \cdot \frac{V}{V}
$$

ce qui porte à :

$$
R_{\mathrm{b}}=50\left(1+m^{\prime} \frac{V^{\prime}}{V}\right)
$$

D'après les règles indiquées dans la circulaire, on est amené à prendre pour $m$ les valeurs du tableau III. Les charges $N$ à faire supporter à la colonne seront données par la formule:

$$
N=\mathrm{R}_{\mathrm{b}}(625+m \omega)
$$

On a ainsi le tableau III.

Les chiffres de la colonne 9 sont obtenus par la formule [B']. Ceux de la colonne $\mathrm{I}$, en divisant la valeur des charges de rupture (tableau I, colonne 4) par les chiffres de la colonne ro. Et, ici, on voit que les coefficients de sécurité effectifs ont une constance remarquable; ce qui permet d'être plus hardi sur la fatigue théorique maxima à admettre.

III et IV. - Exécution des travaux et épreuves des ouyrages. - Les instrucions sur ces deux matières se justifient d'elles-mêmes, et il n'y a pas à s'y arrêter ici.

En résumé, la Commission a fait son possible pour donner aux ingénieurs des instructions aussi précises que le comporte le sujet, à éclaircír ces instructions en tant que de besoin par la circulaire à y joindre, et à faciliter les calculs de résistance à ceux des ingénieurs qui le désirent, le tout sans empiéter en rien sur leur libre arbitre, lequel doit rester, ici, plus absolu que partout ailleurs, puisqu'il s'agit d'une province nouvelle dans l'art de bâtir, qui s'offre à leurs études et à leur activité, et dans laquelle, d'ailleurs, plusieurs d'entre eux ont été parmi les premiers pionniers qui ont préparé les voles actuellement suivies.

L'Inspecteur général, Président et rapporteur, de la Commission,

(A suive.) Maurice LEvy.

\section{Le Développement de la Traction électrique ${ }^{(*)}$}

Le succès obtenu par les tramways électriques fit songer à des applications plus étendues de ce système de traction. D'une part, le besoin sans cesse croissant de moyens de transport dans les villes rendait nécessaire la construction où l'extension des chemins de fer métropolitains, aé. riens ou souterrains, pour lesquels la traction électrique était tout indiquée; d'autre part, on songeait-à établir de nouvelles jonctions interurbaines qui, pour certains réseaux particuliers, se prètaient mal à l'exploilalion par locomotives á vapeur; enfin, on sentait la nécessité d'électrifier certaines portions de quelques grandes lignes do chemins de fér établies én tunnel ou en soulerrain.

Parmi les avantages les plus importants qu'offre la traction électrique, il faut citer la possibilité d'utiliser pour la propulsion des trains l'énergie des chutes d'eau, et de n'ê. tre plus tributaire des mines de charbon de l'étranger. Cet avantage avait été nettement senti par le gouvernement italien qui, dès 1900, entrait résolument dans la voie de l'électrification des chemins de fer, et commençait par écuiper, dans le nord de l'Italie, deux lignes de plus de 100 kilomètres de longueur. L'une, reliant Milan à Gallarate et Varèze, sur le lac Majeur, était équipée à courant continu avec prise de courant par troisième rail. L'autre, celle de Lecco à Sandrio et Chiavenna, par la vallée de la Valtelline, fonctionne avec du courant alternatif triphasé à 3000 volts, 16 périodes.

En 1902-1903, l'Allgemeine Elettricitäts Gesellschaft procédait aux célèbres expériences de Zossen, qui ont été décrites dans La Houille Blanche de janvier 1904. Enfin, á l'heure actuelle, le nombre des lignes à traction électrique à long parcours, et à grand trafic, devient de plus en plus élevé.

La traction électrique présente, sur la traction à vapeur, certains avantages importants. Ces avantages se manifestent par un accroissement considérable de trafic sur les lignes électrifiées. On peut citer de nombreux exemples de cel accroissement, et, d'après $M$. de Valbreuse, sur le chemin de fer de Manhattam à New-York, le trafic a augmenté de 50 pour 100 dans la première année, alors que les frais d'exploitalion tombaient de 55,79 à 40,20 pour 100 des recettes brutes. Sur la ligne Milan-Gallarate-Varèze, l'accroissement de trafic a été de 170 pour 100 en trois ans. Ce résul. tat provient surtout de ce que l'emploi de la traction électrique permet une méthode rationnelle d'exploitation, avec des convois fréquents et relativement légers; les trains sont

( $\left.{ }^{\star}\right)$ Résumé d'une conférence faite le 9 janvier 1907 à la société Internationale des Electriciens par M, de YaLBREuse, et de la discussion qui s'en est suivie, lors des séances des 6. février et 10 avril. 